\title{
Factores que influyen en el comportamiento del consumidor turista: el caso de Manzanillo, México
}

Ernesto Manuel Conde Pérez Carlos Mario Amaya Molinar Edna Alejandra González Alatorre Universidad de Colima

\section{Resumen}

Se contrastan los planteamientos académicos sobre el comportamiento del consumidor y la gestión de la mercadotecnia de destino turístico: se toma como caso de estudio el puerto de Manzanillo, México, y se analiza la evolución de las estadísticas oficiales sobre la afluencia de turistas, su derrama económica, la tasa de ocupación y el impuesto para la promoción, en el pasado reciente. Las conclusiones del estudio sugieren que los resultados positivos en la atracción de visitantes obedecen más a los atributos naturales del destino y a su ubicación que a la calidad de la operación turística por los prestadores de servicios del ramo y a la gestión de la mercadotecnia por la oficina de convenciones y visitantes del destino.

Palabras clave

Manzanillo, consumidor, destino turístico, turista, mercadotecnia 


\title{
Factors that influence consumer-tourist behavior: the case of Manzanillo, Mexico
}

\author{
Ernesto Manuel Conde Pérez \\ Carlos Mario Amaya Molinar \\ Edna Alejandra González Alatorre \\ Universidad de Colima
}

\begin{abstract}
The port of Manzanillo, Mexico, is taken as a case study. This study contrasts academic expositions on consumer behavior and the steps taken for marketing a tourist destination. The evolution of official statistics on the influx of tourists, the effect on the economy, the occupancy rate and the tax for promotion in the recent past are analyzed. The conclusions of this research suggest that the positive results in the attraction of visitors are due to the natural attributes of the destination rather than the quality of services by tourist operators, service providers, and marketing negotiations performed by the local conventions and visitors office.
\end{abstract}

Key WORDS

Manzanillo, consumer, tourist destination, tourists, marketing 


\section{Introducción}

La literatura académica contemporánea ha acumulado una considerable cantidad de trabajos sobre la mercadotecnia de destinos turísticos y la secuencia teórica general: imagen > calidad > valor percibido > satisfacción > lealtad, propuesta por Kotler, Bowen y Makens (1993). Entre los estudios de las relaciones entre imagen, calidad, valor percibido, satisfacción y lealtad aplicados a servicios y destinos turísticos encontramos los trabajos de Baker y Crompton (2000), Baloglu (2000), Bowen y Chen (2001), Bigné, Sánchez y Sánchez (2001); Yang y Peterson (2004); Lee, Yoon y Lee (2007); Chen y Tsai (2007); Yuan y Jang (2008); Simpson y Siguaw (2008); Chi y Qu (2007); Williams y Soutar (2009), y Chen y Chen (2010).

Otro conjunto de autores estudia la gestión de los destinos turísticos; entre ellos Valls et al. (2004), Pike (2004), Ritchie y Crouch (2005), la Organización Mundial del Turismo (омт, 2007, 2010), Miralbell (2007) y Elbe, Hallén y Axelsson (2009). También hay trabajos que examinan la mercadotecnia de destinos turísticos, en ellos se analizan temas relativos a su promoción, segmentación de mercados, posicionamiento y gestión de marcas: Crompton (1992), Pike (2005, 2010), Murdaugh (2005), la Organización Mundial del Turismo y la Comisión Europea de Viajes (2009), Conde et al. (2010) y Gartner y Konecnik (2011).

En la literatura académica mencionada no se encontró ningún estudio que compare los planteamientos teóricos sobre la gestión de la mercadotecnia de destinos turísticos y el comportamiento del consumidor. Así, en este trabajo se analiza un conjunto de elementos que influyen en la decisión del consumidor turista para visitar el destino Manzanillo, atendiendo las acciones de la oficina de gestión de ese puerto en materia de promoción y las percepciones del consumidor sobre atributos como la calidad, el valor percibido, la atención del personal de servicio, la actitud de los habitantes de la localidad, las experiencias de visitas previas y las recomendaciones de familiares y amigos. 


\section{La mercadotecnia de los destinos turísticos}

\section{Comportamiento del consumidor en los destinos turísticos}

La secuencia teórica general: imagen $>$ calidad $>$ valor percibido $>$ satisfacción > lealtad, propuesta por Kotler, Bowen y Makens (1993) ha sido aplicada por diversos académicos en el campo de la investigación del comportamiento del consumidor en destinos turísticos.

El estudio de la imagen de los destinos turísticos resulta relevante por el papel de esta en el comportamiento de consumo de los visitantes, desde el proceso de elección del destino hasta que, al concluir su viaje, deciden regresar y recomendarlo o no a sus familiares y amigos. Pike (2002, 2009) identifica 142 artículos publicados entre 1973 y 2000 que estudian la imagen de los destinos turísticos, a los que agrega otros 28 sobre gestión de marcas de destinos turísticos.

La imagen de destino turístico se entrelaza con la satisfacción del visitante, la percepción de la calidad de los servicios consumidos y las intenciones conductuales de los visitantes. Crompton (1979), Kotler y Gertner (2004) definen sucintamente la imagen como la suma de creencias, ideas e impresiones que una persona tiene de un destino turístico. Pike (2004), Bigné, Sánchez y Sánchez (2001) remiten los componentes cognitivos, afectivos y conativos de la imagen de un destino turístico a asociaciones mentales.

Por lo general, los académicos del área desagregan la imagen del destino turístico en componentes particulares para investigarla. Gallarza, Gil y Calderón (2002) elaboraron una lista con los 20 atributos de la imagen de destino más utilizados por los estudiosos. Frente a la tendencia de los investigadores a descomponer la imagen de destino turístico, Echtner y Ritchie (1991) pasaron de identificar y medir las percepciones de atributos aislados a integrar una imagen general, incluyendo componentes cognitivos, afectivos y conativos, y señalaron que las impresiones holísticas se basan en las combinaciones e interacciones de los atributos de un destino turístico, mismas que resultan influidas por impresiones generales y sentimientos, planteamientos que Baloglu y McCleary (1999) apoyan.

Los trabajos sobre comportamiento del consumidor en destinos turísticos de Barroso, Martín y Martín (2007); Bigné, Sánchez y Sánchez (2001); Chen y 
Tsai (2007), Chi y Qu (2007) y Chen y Chen (2010) confirman empíricamente la secuencia teórica elaborada por Kotler, Bowen y Makens (1993). La imagen influye en la percepción de la calidad por los clientes: una imagen más positiva se corresponde con una mayor calidad percibida, la cual, a su vez, determina la satisfacción de los consumidores, pues la satisfacción es el resultado de la evaluación de la calidad por los clientes. Parasuraman, Zeithaml y Berry (1988) definen la calidad como el juicio general del consumidor sobre la excelencia del servicio y, más específicamente, como un tipo de actitud, relacionada con la satisfacción, descrita como el grado y la dirección de las discrepancias entre las expectativas del servicio y la percepción del desempeño.

Las definiciones de la satisfacción del consumidor tras la visita a un destino turístico de Chon (1989), Chen y Tsai (2007), Chi y Qu (2008), Simpson y Siguaw (2008) y Williams y Soutar (2009) enfatizan el cumplimiento de expectativas y el carácter holístico de la experiencia de visita al destino. Chon (1989) se refiere al papel de la imagen de destino turístico en la satisfacción del visitante, y afirma que se basa en la adecuación entre sus expectativas sobre el destino turístico y la evaluación de la percepción de la experiencia de visita.

Para Baker y Crompton (2000) la satisfacción general del consumidor con una transacción es una función de su evaluación de la calidad del servicio, la calidad del producto y el precio, según el precepto "la calidad lleva a la satisfacción”. Ritchie y Crouch (2005) plantean que las personas que visitan un destino turístico buscan experiencias memorables con calidad total; así, los destinos turísticos deben ser proveedores de experiencias placenteras e inolvidables para los visitantes, con el fin de producir elevados niveles de satisfacción que generen comentarios favorables.

Siguiendo el modelo clásico, Yuan y Jang (2008) estiman que la calidad es el antecedente de la satisfacción y de las intenciones conductuales del consumidor, con la mediación del valor percibido. Hu, Kandampully y Juwaheer (2009) afirman que los consumidores distinguen entre organizaciones competidoras por la calidad en el servicio, por lo que su búsqueda resulta imperativa para las instituciones interesadas en mejorar su competitividad, como un factor que contribuye a incrementar la participación de mercado y la satisfacción del consumidor. 
En su trabajo sobre capital de marca de Eslovenia, Konecnik y Gartner (2007) consideran que la calidad impacta decisivamente en el comportamiento del consumidor y en sus intenciones de visitar, regresar o recomendar el sitio. Pike et al. (2009) han definido la calidad percibida como la percepción de superioridad de un producto o servicio en relación con alternativas relevantes y con su propósito fijado; su trabajo identifica una relación positiva entre percepciones de calidad, imagen de marca y lealtad a la marca.

Los hallazgos presentados en los trabajos de Caruana, Money y Berthon (2000), Sánchez et al. (2006), Chen y Tsai (2007), Williams y Soutar (2009), Boo, Busser y Baloglu (2009), Okello y Yerian (2009), y Chen y Chen (2010) demuestran el papel del valor percibido ${ }^{1}$ para conseguir la calidad percibida, la satisfacción y la lealtad del consumidor, identificando una relación positiva entre el valor percibido de la marca de un producto y las intenciones conductuales futuras de los consumidores.

Caruana, Money y Berthon (2000) concluyen que la satisfacción no depende solo de la calidad en el servicio, y que elevados niveles de calidad resultan valiosos en la medida en que los consumidores piensen que el valor percibido se incrementa, pues un precio competitivo puede contribuir, a través del valor percibido, a buenos niveles de satisfacción, aunque el servicio consumido no tenga elevados niveles de calidad. En ese sentido, Chen y Tsai (2007); Lee, Yoon y Lee (2007); Chen y Chen (2010), y Okello y Yerian (2009) encontraron que la calidad del viaje tiene un efecto más indirecto que directo en la satisfacción general, pues se ve moderada por el valor percibido, lo cual implica que, a menos que conduzca a un incremento en el valor percibido, la calidad del viaje no garantiza la satisfacción general del cliente, por lo que las intenciones conductuales también serán inciertas.

Oliver (1999) analiza las relaciones entre la satisfacción y la lealtad, y establece que estas no operan en paralelo, pues la primera no necesariamente conduce a la segunda, pero sí constituye un antecedente indispensable para que exista la segunda; su ausencia erosiona de modo sensible la adhesión de los consumidores. La lealtad del consumidor resulta primordial para las empresas, por el gran impulso que confiere a su marketing (mercadotecnia), reduciendo

${ }^{1}$ Definido como una variable multidimensional que modera las relaciones entre la calidad y la satisfacción del consumidor. 
los costos de adquisición y atención a la clientela, y disminuyendo su sensibilidad hacia los precios. Los clientes leales pueden ser considerados una importante fuerza de promoción que aporta recomendaciones, divulga comentarios positivos, genera referidos y realiza la mejor publicidad que una empresa puede obtener, por lo que prácticamente se comportan como socios comerciales, todo lo cual repercute en incrementos sustanciales en la captación de ingresos y en la rentabilidad. La satisfacción del consumidor, si bien resulta un antecedente indispensable, no equivale a su lealtad.

En el campo de la mercadotecnia de destinos turísticos, Chen y Tsai (2007) definen las intenciones conductuales como el juicio del consumidor sobre la probabilidad de regresar al destino o de recomendarlo a otros. Oppermann $(1999,2000)$ destaca la relevancia de la lealtad de los visitantes para los destinos turísticos, pues muchas atracciones y sitios dependen de manera considerable de visitantes repetidores.

Para Boo, Busser y Baloglu (2009) la lealtad es el apego del consumidor a una marca, considerándola la dimensión clave del capital de marca de destinos turísticos, y señalando que el capital de marca deriva de la mayor confianza que los consumidores depositan en una marca frente a las competidoras, lo cual se traduce en lealtad y hasta en la disposición para pagar precios mayores por la marca elegida, por lo que la generación de la lealtad de los consumidores constituye un objetivo esencial en el branding. Al igual que Konecnik y Gartner (2007), Boo, Busser y Baloglu (2009) clasifican la lealtad en conductual y actitudinal.

\section{Oficinas de gestión de destinos turísticos}

La responsabilidad de la comercialización de un destino turístico recae en las oficinas de gestión de destinos turísticos o DMo; ${ }^{2}$ son las instancias encargadas de organizar, coordinar y dirigir los esfuerzos de los diversos actores públicos y privados que intervienen en la operación de un destino turístico. Elbe, Hallén y Axelsson (2009: 288) definen las DMo como "cualquier organización de cualquier nivel responsable del marketing de un destino turístico definido”, o como "una entidad que debe coordinar los esfuerzos para atraer visitantes a su domi-

${ }^{2}$ Destination management organization. 
nio geográfico”. Para Miralbell (2007), las Dmo son el lugar y servicio donde los actores y agentes del sector turístico del destino se coordinan, uniendo sus estrategias para competir. Por su parte, Cals, Capella y Vaqué (1997) las conciben como las dependencias creadas por una administración pública, directamente o en colaboración con otras entidades, para gestionar todos los servicios relacionados con la recepción de visitantes y organizar los trabajos de coordinación, promoción y comercialización de productos turísticos de su demarcación.

Ritchie y Crouch (2005) consideran que la DMo es el organismo encargado de: a) coordinar y dirigir los esfuerzos de los diversos integrantes del sistema turístico, b) contar con el apoyo de los principales actores del sistema y c) disponer de las herramientas necesarias para fomentar el adecuado desarrollo de la oferta turística. A su vez, Bornhorst, Ritchie y Sheehan (2010) afirman que las funciones esenciales de la DMo son trabajar con eficiencia para mejorar los niveles de bienestar de los habitantes del destino turístico y asegurar que los visitantes experimenten vivencias altamente satisfactorias y memorables, ofrecidas a precios razonables, a fin de que el destino opere de forma sostenible en sus vertientes económica, social, cultural y ambiental.

Para Pike (2004), las Dmo son organizaciones dedicadas a la mercadotecnia de los destinos, y su objetivo esencial es mejorar la competitividad sostenible del destino turístico, lo cual se logra mediante cuatro propósitos particulares: mejorar la imagen del destino, incrementar la rentabilidad de su industria turística, reducir la estacionalidad de los flujos de visitantes y asegurar el financiamiento en el largo plazo.

Valls et al. (2004) señalan que las modalidades de organización que pueden asumir las DMo son muy variadas; cada país, región o destino del mundo puede diseñar sus Dмo a su medida o conveniencia, pues los diferentes actores que intervienen en la actividad turística pueden ponerse de acuerdo y organizarse como mejor les acomode: algunas se integran por membresía individual o se componen de organismos y cámaras empresariales, como asociaciones de hoteleros y restauranteros; otras DMo asumen la figura de oficina de convenciones y visitantes (ocv); algunas más son estructuras netamente gubernamentales, aunque la tendencia actual es que presenten estructuras mixtas, con participación público-privada. 
Pike (2004) subraya que no existe un modelo universalmente aceptado para integrar las Dmo, pues exhiben una "plétora" de modalidades de estructuras organizacionales, pese a que la gran mayoría se organiza mediante organismos de participación público-privada. La estructura que asuma la DMo tendrá un importante impacto en su financiamiento: aquellas con estructuras gubernamentales dependerán sobre todo de impuestos al consumo turístico o de recursos fiscales, mientras que las conformadas por membresías de operadores turísticos dependerán básicamente de las cuotas de los socios.

Presenza, Sheehan y Ritchie (2005) proponen un modelo conceptual con dos funciones fundamentales de la DMo: hacia el exterior, la mercadotecnia del destino; hacia el interior, el desarrollo del destino. Consideran que la competencia crítica más importante de una Dmo es la coordinación de los agentes de riesgo. En su estudio sobre 25 destinos turísticos canadienses, Bornhorst, Ritchie y Sheehan (2010) concluyeron que los asuntos más relevantes para los entrevistados son la mercadotecnia del destino turístico, las relaciones con los agentes de riesgo y con la comunidad y los resultados en los indicadores de desempeño de la Dmo y del destino.

Así, el análisis de los planteamientos teóricos y empíricos sobre las funciones de una Dмo arroja una gran diversidad de criterios, aunque parece existir consenso en que las funciones primordiales son la mercadotecnia del destino y la coordinación de los diversos agentes de riesgo interesados. También parece haber acuerdo respecto de la importancia de la atención a los visitantes, la gestión de la calidad de la experiencia de destino y el fomento económico. Sin embargo, después de estos cuatro temas, el acuerdo sobre las principales funciones de una Dmo tiende a disolverse.

\section{La mercadotecnia de destinos turísticos}

Algunos académicos limitan el tema de la mercadotecnia de destinos turísticos a acciones operativas, como la formulación del plan de mercadotecnia del destino, la segmentación y la instrumentación de la mezcla promocional, y tratan por separado los temas relativos a la gestión de marca, la imagen del destino y el posicionamiento. 
Para definir la mercadotecnia de destinos turísticos, Pike (2004) y Murdaugh (2005) citan como punto de partida la definición de mercadotecnia formulada por Kotler, Bowen y Makens (1993: 12), ampliamente aceptada en el campo académico disciplinar: "La mercadotecnia es un proceso gerencial y social mediante el cual los individuos y los grupos obtienen lo que necesitan creando e intercambiando productos y valor con otros". Diversos textos de mercadotecnia de destinos turísticos incluyen entre sus temas particulares la gestión de marca, la imagen, el posicionamiento y el comportamiento del consumidor; para los fines del presente trabajo, se adopta esta visión más extensa de la mercadotecnia de destinos.

Murdaugh (2005) considera que la tarea de mercadotecnia de la Dmo es incrementar gradualmente la cantidad de visitas al destino turístico y sus beneficios económicos entre la comunidad, para lo que debe concentrarse en las funciones de investigación de mercado, publicidad, gestión de marcas, planeación estratégica de la mercadotecnia y mercadotecnia por internet. En este contexto, ubica a la mercadotecnia del destino como la función más importante de las DMo, a las que considera como organismos generales encargados de la promoción del conjunto de empresas que ofrecen servicios al turista. Menciona tres criterios básicos para evaluar las acciones de mercadotecnia de una Dmo: arrojar beneficios económicos palpables en el destino, diseñar y ejecutar con éxito las iniciativas de mercadotecnia y reportar sus acciones y resultados con transparencia.

Pike (2004) también se refiere a la mercadotecnia de destinos turísticos enfatizando las actividades operativas y la mezcla promocional. Ritchie y Crouch (2005) añaden a la discusión entre organizaciones de gestión de destinos turísticos y mercadotecnia de destinos un debate entre mercadotecnia y promoción, pues aseguran que gran parte de los gerentes de destino equiparan ambos conceptos.

\section{Metodología}

Para conocer los factores que influyen en el comportamiento del consumidor turista de un destino se propone una investigación descriptiva orientada a determinar los siguientes aspectos: 
- Caracterización del destino turístico: tener presente la ubicación y el clima del destino, la cantidad de turistas hospedados en los hoteles, la evolución de la afluencia de visitantes, la estancia y gasto promedio diario, la evolución de la oferta de hospedaje y la tasa de ocupación y la derrama económica por concepto de turismo.

- Perfil de los encuestados: en particular la procedencia de quienes visitan el destino, su edad, grado de escolaridad, situación laboral y económica.

- Motivaciones y características del viaje: los motivos por lo que se elige el destino turístico en cuestión.

- Imagen del destino, lealtad e intenciones conductuales: valorar los atributos del destino.

Para la investigación de campo se utilizó un cuestionario dividido en cuatro apartados, según se muestra en el cuadro 1. Los ítems orientados a la valoración de los atributos del destino por los encuestados se construyeron utilizando una escala de Likert de cinco puntos.

\section{Cuadro 1. Estructura del cuestionario}

$\begin{array}{lll}\text { Bloque } & \text { Ítems } & \text { INFORMACIÓN SOLICITADA } \\ \text { I } & 1-16 & \begin{array}{l}\text { Perfil del visitante, motivo del viaje, duración, grupo de via- } \\ \text { je, transporte, hospedaje. }\end{array} \\ \text { II } & 17-19 & \begin{array}{l}\text { Fuentes de información sobre el destino. } \\ \text { III }\end{array} \\ \text { 20-24 } & \begin{array}{l}\text { Situación económica, ingreso disponible para viajar, percep- } \\ \text { ción de los precios de servicios. }\end{array} \\ \text { IV } & 25-30 & \text { Evaluación de atributos del destino, intenciones conductuales. }\end{array}$

Fuente: Elaboración propia.

El universo de estudio se calculó utilizando el método de muestreo probabilístico por estratos, mediante la fórmula estipulada por la Secretaría de Turismo en el fascículo V, Competitividad y Desarrollo de Productos Turísticos Exitosos, de la serie de documentos técnicos de competitividad: 
Fórmula para la población infinita (más de 100 000)

$$
n=\div \frac{(3.84 * p * q)}{E^{2}}
$$

Fórmula para la población finita (menos de 100 000)

$$
n=\div \frac{(3.84 * N * p * q)}{E^{2}(N-1)+(3.84 * p * q)}
$$

Donde:

$\mathrm{n}=$ es la muestra que se desea obtener.

$p=50 \%$ (o participación del segmento)

$q=50 \%$ (o 1menos la participación del segmento)

$n=$ tamaño muestral

$N=$ Universo o población (total de turistas captados por la región)

$E=$ error de la estimación que representa el .05, nivel de confianza de $95 \%$

3.84 es un valor constante obtenido de la tabla de áreas bajo la curva normal tipificada de 0 a $Z$, con base en el valor máximo de error.

Los cuestionarios se aplicaron en diversos sitios públicos: el Aeropuerto Internacional de Manzanillo, hoteles, establecimientos de alimentos y bebidas, transportes públicos y en el centro histórico de Manzanillo, y se efectuaron en total 384, de los cuales 319 resultaron válidos, es decir $81.77 \%$. De los cuestionarios realizados, $92 \%$ fue respondido por turistas nacionales y $8 \%$ por extranjeros, norteamericanos en su totalidad.

Para verificar la validez y fiabilidad de los constructos incluidos se utilizó el coeficiente alpha de Cronbach, con el que se obtuvo un promedio de 0.83 para el conjunto de los ítems del cuestionario; con este procedimiento se valora la consistencia interna de las escalas de Likert diseñadas y se asegura que efectivamente representen lo que se busca medir. 


\section{Análisis de los resultados}

\section{Caracterización del destino turístico}

Manzanillo es el municipio más extenso y la zona turística más importante del estado de Colima. Cuenta con una población de 161420 habitantes y una extensión territorial de 1578.4 km² (INEGI, 2011). Limita al Sureste con el municipio de Armería, al Sur con el Océano Pacífico, al Noreste con el municipio de Coquimatlán, al Norte con el de Minatitlán, al Noreste y Oeste con el estado de Jalisco. Su clima se clasifica como cálido sub-húmedo y su temperatura media anual es de $26.9^{\circ} \mathrm{C}$, con temporada de lluvias en verano entre junio y octubre (INEGI, 2011).

Por sus condiciones naturales, Manzanillo cuenta con sitios apropiados para la actividad turística, esencial para el desarrollo económico del municipio y la entidad. Sus principales atractivos turísticos son sus playas, alrededor de las cuales se ha construido una gran infraestructura turística, compuesta por hoteles de diversas categorías, marinas, campos de golf, restaurantes y centros nocturnos; asimismo, cuenta con una carretera de cuatro carriles que lo conecta con la capital del estado y con la ciudad de Guadalajara; también operan un aeropuerto internacional y una terminal de cruceros.

Entre sus ventajas comparativas más notables se encuentra el hecho de ser el destino de playa más cercano a Guadalajara, Jalisco, la segunda ciudad más importante de México, con más de cuatro millones de habitantes; cuenta con un clima soleado muy estable durante todo el año, lo cual resulta muy conveniente para el disfrute de las playas en cualquier temporada.

El municipio enfrenta un dilema por la prioridad brindada al desarrollo industrial y portuario frente al desarrollo turístico (Amaya et al., 2011). Las características de sus bahías, muy apropiadas para el resguardo de embarcaciones, hicieron que el municipio se orientara al desarrollo portuario, al grado de convertirse en el puerto más importante de México, por el volumen de comercio con los países de la cuenca del Pacífico, con América del Norte y del Sur y con el Lejano Oriente; también se han instalado en el puerto grandes industrias de las áreas de generación de energía, gas natural, pesca y acero. Evidentemente, no resulta fácil compaginar el turismo con la actividad portuaria e industrial, aunque se presentan oportunidades para el turismo de negocios. 
Factores que influyen en el comportamiento del consumidor turista: el caso de Manzanillo

Cuadro 2. Turistas hospedados en hoteles en Manzanillo, 2010-2012

\begin{tabular}{llll}
\multirow{2}{*}{ Año } & \multicolumn{3}{c}{ TuRISTAS } \\
\cline { 2 - 4 } 2010 & Nacionales & Extranjeros & Total \\
2011 & 623443 & 29823 & 653266 \\
2012 & 689781 & 30894 & 720675 \\
\hline
\end{tabular}

Fuente: Gobierno del Estado de Colima, 2010, 2011, 2012.

De acuerdo con la información oficial presentada por la Secretaría de Turismo del Estado de Colima (informes de gobierno del licenciado Mario Anguiano Moreno, 2010, 2011, 2012), Manzanillo recibe alrededor de 70 \% de los turistas que visitan el estado. Entre 2010 y 2012, su afluencia de turistas se incrementó en poco más de 100000 viajeros, lo que representa un crecimiento promedio para el periodo de $8.5 \%$ (gráfica 1). Solo $4 \%$ de los turistas hospedados en Manzanillo en el periodo mencionado son extranjeros, provenientes sobre todo de Estados Unidos y Canadá. La procedencia nacional de la mayoría de los visitantes brinda estabilidad a su demanda turística.

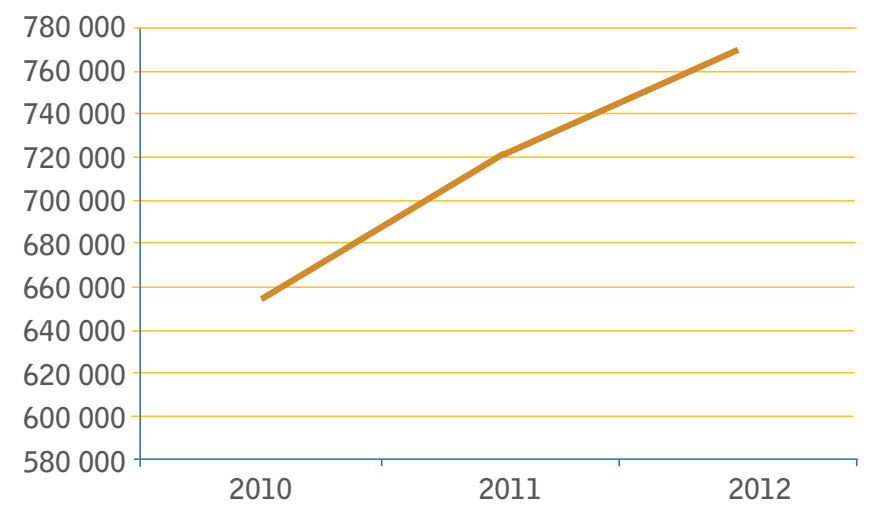

Fuente: Gobierno del Estado de Colima, 2010, 2011, 2012.

Gráfica 1. Evolución de la afluencia de turistas en Manzanillo, 2010-2012 
El cuadro 3 muestra que la estancia promedio de los turistas que estuvieron en Manzanillo entre 2010 y 2012 fue de aproximadamente dos días y medio; se posiciona como un destino turístico de fin de semana o de vacaciones cortas, con lo que se explican sus bajos niveles de ocupación, a pesar de los considerables incrementos en la cantidad de visitantes registrados en el lapso analizado; el gasto promedio por turista asciende a cerca de 321 dólares, 25 \% menos que el gasto promedio por turista internacional reportado por el Consejo Nacional Empresarial Turístico para el 2011 (CNET, 2012), equivalente a 427 dólares por turista.

Cuadro 3. Estancia y gasto promedio diario en Manzanillo, 2010-2012

\begin{tabular}{lcc} 
AÑo & Estancia Promedio (días) & Gasto PROMEdio diario (MXP) \\
\hline 2010 & 2.34 & $\$ 1547$ \\
2011 & 2.55 & $\$ 1875$ \\
2012 & 2.5 & $\$ 1741$ \\
\hline
\end{tabular}

Fuente: Gobierno del Estado de Colima, 2010, 2011, 2012.

Cuadro 4. Evolución de la oferta de hospedaje y la tasa de ocupación en Manzanillo, 2010-2012

\begin{tabular}{rcccc} 
AÑo & Hoteles & CuARTOS & CAMAS & \% de ocupación \\
2010 & 122 & 5172 & 9258 & 45.70 \\
2011 & 117 & 5142 & 10052 & 48.75 \\
2012 & 115 & 5115 & 9954 & 45.56 \\
\hline
\end{tabular}

Fuente: Gobierno del Estado de Colima, 2010, 2011, 2012.

En el cuadro 4 puede apreciarse cómo la oferta de hospedaje de Manzanillo se ha reducido entre 2010 y 2012 en términos de establecimientos, aunque la oferta de habitaciones y camas no presenta variaciones considerables; sin embargo, durante el periodo mencionado, la tasa de ocupación ha permanecido relativamente estable, en niveles que los estudiosos de la economía turística consideran muy por debajo del nivel de rentabilidad, ubicado en un umbral inferior promedio de 65 \% de ocupación (Lundberg, Krishnamoorthy y Stavenga, 1995). 
Factores que influyen en el comportamiento del consumidor turista: el caso de Manzanillo

Cuadro 5. Impuesto al hospedaje para la promoción turística de Manzanillo, 2010-2012

\begin{tabular}{lll} 
AÑo & IMPUESTOS SOBRE HOSPEDAJE & USD \\
2010 & 6760605 & 512167 \\
2011 & 6537299 & 495250 \\
2012 & 10171364 & 770558 \\
\hline
\end{tabular}

Fuente: Gobierno del Estado de Colima, 2010, 2011, 2012.

Como puede observarse en el cuadro 5, el impuesto al hospedaje recaudado por el Fideicomiso de Turismo de Manzanillo, operado por la oficina encargada de la gestión turística del destino (la ocv de Manzanillo), se incrementó 50 \% entre 2010 y 2012, lo cual obedece básicamente a que en 2012 aumentó en esa proporción el impuesto al hospedaje en el estado de Colima, pasando de 2 a $3 \%$.

Cuadro 6. Derrama económica por concepto de turismo en Manzanillo, 2010-2012

\begin{tabular}{ll} 
AÑO & DERRAMA ECONÓMICA (PESOS) \\
2010 & 2935607477 \\
2011 & 3619483029 \\
2012 & 4054114939 \\
\hline
\end{tabular}

Fuente: Gobierno del Estado de Colima, 2010, 2011, 2012.

El cuadro 6 muestra las cifras oficiales sobre derrama económica en Manzanillo entre 2010 y 2012; de acuerdo con estadísticas de la Secretaría de Turismo del Estado de Colima, la derrama por turismo se incrementó 38 \% , proporción que resulta exorbitante frente a $8.5 \%$ de crecimiento en la cantidad de visitantes, con $11.5 \%$ en el crecimiento de turistas extranjeros y $12.5 \%$ en el gasto promedio diario, con una inflación para el periodo de $11.05 \%$ (INEGI, 2013) y decrementos en el porcentaje de ocupación y en la cantidad de establecimientos de hospedaje y habitaciones. 


\section{Perfil de los encuestados}

Los turistas nacionales encuestados ( $92 \%$ del total de la muestra) procedían principalmente de los estados de Colima, Jalisco, Distrito Federal y Michoacán, ubicados en las zonas centro y occidente del país. En cuanto al turismo internacional, 81 \% por ciento de los encuestados era originario de Estados Unidos, y $18 \%$ de Canadá.

Las preguntas fueron contestadas principalmente por jóvenes, con edades entre 18 y 30 años (72\% de los encuestados); los turistas con edades comprendidas entre los 31 y los 41 años representaron $17 \%$ de la muestra, y los visitantes con edades entre 42 y 52 años constituyeron $11 \%$. Del total de las personas que respondieron, $52 \%$ tenía estudios de licenciatura y $35 \%$ de preparatoria; solo $1 \%$ contaba con posgrado. Respecto a su situación laboral, 55 \% estudiaba, $40 \%$ era empleado o trabajador por cuenta propia y $5 \%$ estaba jubilado; $46 \%$ de los encuestados consideraba que su situación económica era buena en relación con años anteriores, 37 \% afirmó que su situación era regular y 6 \% señaló que su economía era mala; 2 \% no contestó.

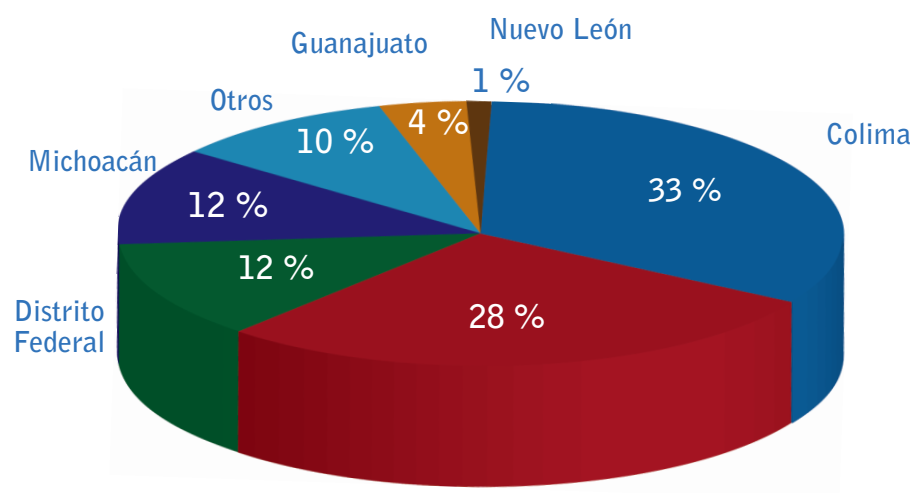

Jalisco

Fuente: Elaboración propia.

Gráfica 2. Procedencia de los turistas nacionales que visitan Manzanillo 


\section{Motivación y características del viaje}

Manzanillo fue elegido por $82 \%$ de los encuestados como su primera opción de viaje, y 80 \% manifestó viajar por vacaciones o placer. Las visitas a familiares o amigos constituyeron la segunda razón para visitar el puerto, con 9 \% de respuestas, mientras que los estudios y los negocios representaron 6 y $5 \%$ del objeto del viaje, respectivamente.

En la gráfica 3 se presentan los motivos mencionados por los encuestados para visitar Manzanillo; en primer lugar, con $56 \%$ de respuestas, destacan las playas, seguidas por las experiencias de visitas anteriores (26\%), la proximidad geográfica (23\%), el clima (19\%) y las recomendaciones de familiares y amigos (17\%).

Más de la mitad de los turistas que acuden a Manzanillo duran hasta tres días en el destino; por lo general, un fin de semana. No obstante, $25 \%$ de la muestra expresó estar una semana, en especial durante las vacaciones de semana santa y de verano; $10 \%$ afirma pasar más de una semana y menos de un

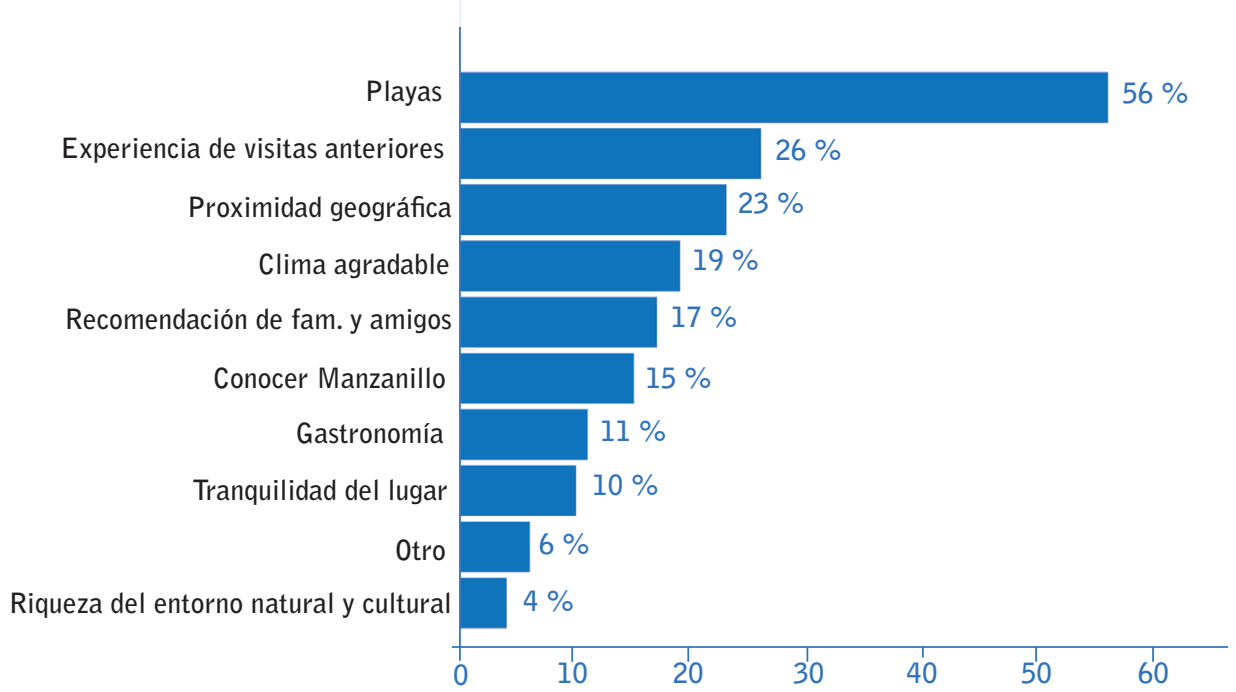

Fuente: Elaboración propia. 


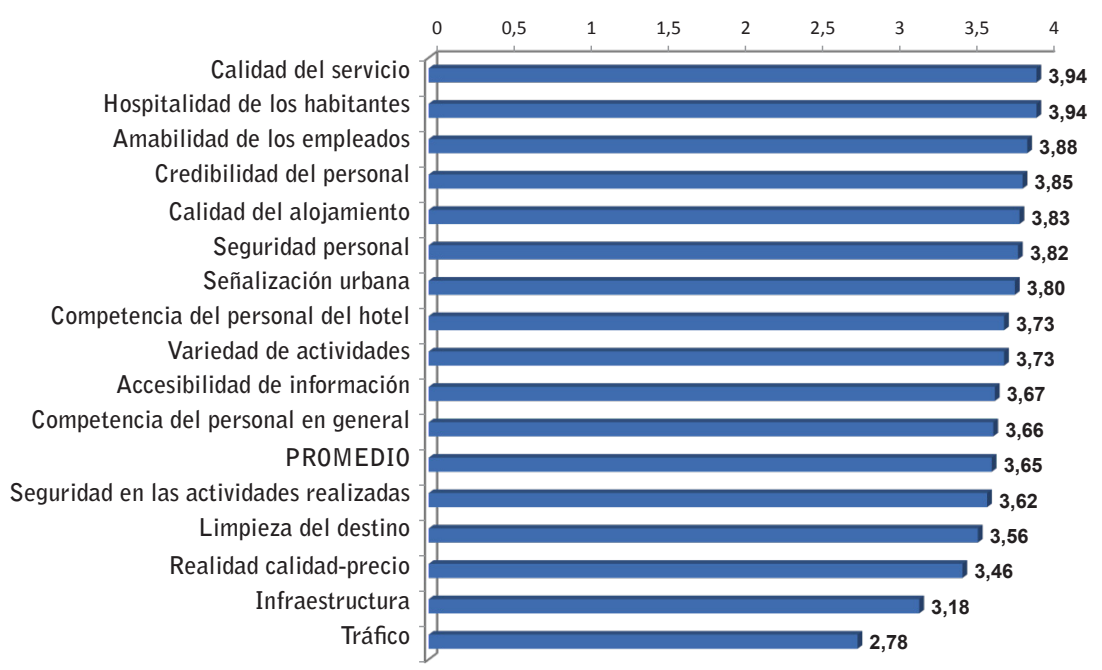

Fuente: Elaboración propia.

Gráfico 4. Valoración de diversos atributos del destino Manzanillo

mes; esta respuesta correspondió casi en su totalidad a turistas procedentes de Estados Unidos y Canadá. En cuanto a la composición del grupo de viaje, 83 \% viaja con familiares y amigos, y $11 \%$ con su pareja, en tanto que $6 \%$ va solo, ya que visita Manzanillo por negocios relacionados con el puerto.

El porcentaje de encuestados que empleó automóvil propio para trasladarse a Manzanillo fue de $73 \%$, principal medio de transporte hacia el destino, seguido por el autobús y el avión. Los visitantes eligieron estas opciones de transporte por comodidad (61 \%), precio (21 \%), tiempo (10 \%) y opción única (8 \%). En cuanto al medio por el cual los turistas se enteraron de Manzanillo, 65 \% señaló que conocieron el destino turístico por recomendación de un familiar o amigo, 22 \% por la publicidad y $4 \%$ por agencias de viaje. Los encuestados consideran que Manzanillo es un destino accesible en cuanto a precios en transporte, hospedaje, alimentación y recreación; solo $25 \%$ de ellos considera que los precios son altos en hospedaje y alimentación. 


\section{Cuadro 7. Valoración de diversos atributos del destino Manzanillo}

\begin{tabular}{clc} 
NúmERO & Atributos & VALOR MEDiO \\
\hline 1 & Calidad en los servicios & 3.94 \\
2 & Hospitalidad de los habitantes & 3.94 \\
3 & Amabilidad de los empleados & 3.88 \\
4 & Credibilidad del personal & 3.85 \\
5 & Calidad del alojamiento & 3.83 \\
6 & Seguridad personal & 3.82 \\
7 & Seguridad urbana & 3.8 \\
8 & Competencia del personal del hotel & 3.73 \\
9 & Variedad de actividades & 3.73 \\
10 & Accesibilidad de información & 3.67 \\
11 & Competencia del personal en general & 3.66 \\
12 & Seguridad en las actividades realizadas & 3.62 \\
13 & Limpieza del destino & 3.56 \\
14 & Relación calidad-precio & 3.46 \\
15 & Infraestructura & 3.18 \\
16 & Tráfico & 2.78 \\
\hline Media & & 3.65 \\
Desviación estándar & 0.30 \\
\hline & & \\
\hline
\end{tabular}

Fuente: Elaboración propia. 


\section{Imagen del destino, lealtad e intenciones conductuales}

Los turistas encuestados valoran los atributos de Manzanillo con una calificación media de 7.3 puntos sobre un total de 10. Los aspectos mejor valorados, en una escala del 1 al 5 (siendo 5 la mejor calificación) son la hospitalidad de los habitantes y la calidad del servicio, con una puntuación media de 3.94, mientras que la amabilidad de los empleados obtuvo una nota de 3.88. El tráfico vehicular recibió la calificación más baja, con 2.78 puntos. En la gráfica 4 se observa que temas como la seguridad en las actividades realizadas, limpieza del destino, relación precio-calidad, infraestructura y tráfico se encuentran por debajo del promedio.

Nueve de cada diez turistas afirmaron que no es la primera vez que viajan al puerto, 54 \% ha visitado Manzanillo más de seis veces; $22 \%$ señaló que es su segunda visita y $14 \%$ ha estado en él entre tres y cinco veces. Solo $10 \%$ resultó visitante de primera vez. Los encuestados que ya conocían Manzanillo mencionaron que, en promedio, lo visitan entre dos y tres veces al año; $22 \%$ acude al destino más de seis veces al año, pues radican cerca; $8 \%$ repite su visita entre cuatro y cinco veces al año.

En lo que se refiere a las intenciones conductuales de los encuestados, 99 \% dijo que piensa regresar a Manzanillo, pues le pareció un destino de sol y playa muy atractivo y, sobre todo, accesible en términos de precios; $94 \%$ de los turistas mencionó que recomendaría la visita a Manzanillo.

\section{Discusión}

En el panorama turístico mundial, el turismo de sol y playa permanece como una de las modalidades de operación turística más consolidadas, en expansión constante y con gran vitalidad. Un sector de la comunidad académica ha criticado duramente este modelo turístico, a pesar de que desempeña un relevante papel para numerosos países y regiones: casi dos tercios de los turistas en México, nacionales o extranjeros, practican el turismo de sol y playa, ya sea en centros de playa integralmente planeados (CIP), desarrollados por el Fondo Nacional de Fomento al Turismo, o en centros de playa tradicionales, como Manzanillo (Presidencia de la República, 2011). 
En las estadísticas sobre visitantes al puerto se percibe un significativo incremento promedio de 8.5 \% entre 2010 y 2012. La situación de Manzanillo coincide con los planteamientos de Pike (2009), para quien los viajes cortos representan una tendencia central en el turismo contemporáneo, frente a un decremento en las vacaciones tradicionales de mayor estancia. En el caso que nos ocupa, casi tres cuartas partes de los viajeros utilizan el automóvil propio como medio de transporte, y proceden del propio estado de Colima o de los estados vecinos -Jalisco y Michoacán.

La evaluación de los atributos del destino turístico por parte de los turistas no puede considerarse sobresaliente, un promedio de 3.65 en una escala de valoración de cinco puntos, equivalente a una calificación general de 7.3. Así, puede afirmarse que la imagen general que produce en sus turistas es llanamente positiva.

En cuanto a los atributos individuales de la imagen del destino sobre habitantes, servicios turísticos y medio ambiente construido, destacan, en el extremo positivo, la percepción de la calidad del servicio y del alojamiento, así como la hospitalidad y la atención del personal y de la población local; en el extremo opuesto se encuentran las percepciones sobre el tránsito vehicular, la infraestructura, la relación calidad-precio y la limpieza del lugar. Además, Manzanillo cuenta con dos atributos naturales que juegan un papel central en la imagen del destino turístico: las playas y el clima.

En los trabajos de Lee, Yoon y Lee (2007): Chen y Chen (2010); Okello y Yerian (2009); Caruana, Money y Berthon (2000); Sánchez et al. (2006); Chen y Tsai (2007); Williams y Soutar (2009); Boo, Busser y Baloglu (2009), que analizan el papel del valor percibido en la calidad, la satisfacción y la lealtad del consumidor, se identifica la relación positiva entre el valor percibido y las intenciones conductuales de los consumidores. En este trabajo, $75 \%$ de los encuestados manifestó que el gasto general del viaje resultó accesible, a lo que puede agregarse que la mayoría de los visitantes son viajeros de proximidad, y que se trasladan por tierra, en automóvil o autobús, dato importante en un país extenso como México, donde viajar por avión es caro, pues no existen las líneas de bajas tarifas. Así, el resultado sugiere que el transporte terrestre a corta distancia incrementa la percepción general de valor percibido entre los turistas.

Los resultados pueden considerarse sobresalientes en las respuestas rela- 
tivas a la lealtad y las intenciones conductuales: $90 \%$ de los encuestados es visitante repetidor y más de $90 \%$ declara sus intenciones de regresar y recomendar a Manzanillo entre sus familiares y amigos, datos que confirman los resultados de trabajos anteriores (Conde et al., 2010) y los planteamientos de Bowen y Chen (2001), Yang y Peterson (2004), Chen y Tsai (2007), Konecnik y Gartner (2007), y Boo, Busser y Baloglu (2009), quienes estudian las intenciones conductuales del consumidor, concibiendo la lealtad actitudinal como un deseo manifiesto de continuar la relación con el proveedor de un servicio, y la lealtad conductual como la proporción de veces que un comprador elige el mismo producto o servicio en una categoría específica. También se comprueban las tesis de Oppermann (1999, 2000), Boo, Busser y Baloglu (2009) y Bowen y Chen (2001), quienes destacan la trascendencia de la lealtad de los visitantes para los destinos turísticos, la perciben como una dimensión clave en su comercialización y definen a los clientes leales como una valiosa fuerza de mercadotecnia que contribuye con recomendaciones y comentarios positivos, generando referidos y publicidad gratuita.

Una explicación a estos significativos resultados puede encontrarse en el hecho de que la principal motivación de los turistas para visitar el destino son las playas, el clima y la proximidad geográfica. Los trabajos de Íñiguez et al. (2007) y de Gutiérrez et al. (2010) ratifican la calidad estética de las playas de Manzanillo, de acuerdo con la norma NMX-AA-120-SCFI-2006, que establece los requisitos y especificaciones de sustentabilidad de calidad de playas; las playas turísticas de Manzanillo cumplen con esta norma durante la mayor parte del año, con excepción de las temporadas pico, como semana santa y algunos puentes, o cuando hay tormentas o huracanes. Como ya se comentó, Baker y Crompton (2000) juzgan que el factor más importante para la satisfacción del consumidor es la calidad: "la calidad lleva a la satisfacción”. A lo anterior, Yuan y Jang (2008) agregan que la calidad percibida antecede a las intenciones conductuales y, en el caso de Manzanillo, una explicación razonable a las positivas intenciones conductuales de los encuestados es que la calidad de sus playas satisface a los turistas, además de su buen clima.

En lo que se refiere a los resultados de la operación de la mercadotecnia del destino turístico por la ocv Manzanillo, podemos ver que ha aumentado la cantidad de visitantes, así como su desmesurada derrama económica, con lo 
que se cumplen los propósitos esenciales planteados por Murdaugh (2005) para una oficina de gestión de destinos turísticos; sin embargo, de acuerdo con lo que propone este autor, no se cumplen los tres criterios básicos necesarios para evaluar las acciones de mercadotecnia de una Dmo: arrojar beneficios económicos palpables en el destino, diseñar y ejecutar exitosamente las iniciativas de mercadotecnia y reportar sus acciones y resultados con transparencia, pues no se conoce la metodología utilizada para calcular los datos presentados en los informes de gobierno, el plan de mercadotecnia de la DMo, ni la forma como se ha ejercido su presupuesto o cómo se ha gastado el impuesto al hospedaje. De esta manera, no es posible saber si se cumplen los lineamientos propuestos por Pike (2004), Murdaugh (2005), Presenza, Sheehan y Ritchie (2005); Ritchie y Crouch (2005) у омт (2007) para la gestión de la mercadotecnia del destino turístico.

Los informes oficiales del gobierno anuncian que entre 2010 y 2012 se recaudó un total de 23469268 pesos mexicanos por impuesto al hospedaje, equivalentes a 1777975 dólares estadounidenses; sin embargo, no se detalla la forma en que se ejercieron, pues solo se enumera un conjunto disperso de acciones y eventos de promoción del destino. Los resultados del presente trabajo arrojan que $65 \%$ de los encuestados conocieron el destino turístico por recomendación de familiares o amigos, mientras que $22 \%$ se enteró por publicidad y $4 \%$ por agencias de viaje; en este último rubro, el informe de gobierno de 2012 (Anguiano Moreno, 2012) menciona que la Dmo participó en 43 eventos distintos, a los que acudieron 8223 agentes de viajes; así sería necesario hablar con 2 055 agentes de viajes y acudir a 10 eventos de promoción para incrementar en $1 \%$ la captación de turistas en Manzanillo; además, de acuerdo con los datos oficiales, entre 2010 y 2012 debió haberse destinado más de un millón de pesos por cada punto porcentual de conocimiento del destino turístico Manzanillo por parte de los turistas.

\section{Conclusiones}

Los resultados demuestran las elevadas intenciones conductuales positivas de los turistas encuestados, aunque también sugieren que, más que una operación turística con excelentes niveles de calidad por parte de las organizaciones públicas y privadas de Manzanillo, los altos niveles de satisfacción de los visitantes 
obedecen a la calidad de sus playas, a su buen clima, a precios accesibles y a que la mayoría de su clientela es viajero de proximidad.

En materia de atributos de su imagen como destino turístico, calidad de los servicios, atención de personal y habitantes, información y señalización, infraestructura, limpieza, etc., dependientes de la intervención de organizaciones públicas y privadas, en general no son sobresalientes.

Las actividades de la oficina de gestión de destinos turísticos resultan una caja negra, pues las autoridades no divulgan sus metodologías de recopilación y procesamiento de información; gran parte de los datos que presentan son inverosímiles y las respuestas de la encuesta sugieren que sus acciones de promoción registran impactos muy reducidos.

Por lo anterior, puede concluirse que la elevada intención conductual de los turistas obedece en gran medida a factores naturales y a la accesibilidad del destino, y no a la operación turística de los prestadores de servicios turísticos o a las acciones de promoción de la ocv Manzanillo.

Las investigaciones académicas sobre la operación turística en el puerto apuntan reiteradamente a limitaciones en las tareas de gestión del destino turístico. Entre las principales restricciones identificadas en la operación de la oficina de gestión puede mencionarse que se concibe a sí misma solo como una oficina de promoción, y deja de lado aspectos como la coordinación con los operadores turísticos y otro tipo de actores locales para mejorar la calidad de los servicios, la limpieza del destino, la información y señalización turística, y la percepción de seguridad, entre otros atributos relevantes para la imagen del lugar.

Otro aspecto que se sugiere mejorar es la transparencia respecto de la formulación de las estadísticas turísticas, la planeación y operación de la mercadotecnia del destino y la gestión del presupuesto de promoción, que aumentó sustancialmente entre 2011 y 2012 (50 \%), sin que ese incremento se haya reflejado en la afluencia de turistas. Por último, las limitaciones evidenciadas en la gestión del destino obedecen a la falta de profesionalización de las autoridades responsables. 


\section{Fuentes consultadas}

Amaya, C. et al. (2011). Gestión del destino turístico Manzanillo. Buenos Aires: El Aleph.

Anguiano Moreno, Mario (2010). Anexo Estadístico del Primer Informe de Gobierno. Mario Anguiano Moreno. Colima: Gobierno del Estado de Colima.

----- (2011). Anexo Estadístico del Segundo Informe de Gobierno. Mario Anguiano Moreno. Colima: Gobierno del Estado de Colima.

(2012). Anexo Estadístico del Tercer Informe de Gobierno. Mario Anguiano Moreno. Colima: Gobierno del Estado de Colima.

Baker, D. y J. Crompton (2000). "Quality, Satisfaction and Behavioral Intentions”. Annals of Tourism Research, 27 (3), 785-804.

Baloglu, S. (2000). "A Path Analytic Model of Visitation Intention Involving Information Sources, Socio-Psychological Motivations, and Destination Image”. Journal of Travel \& Tourism Marketing, 8 (3), 81-90.

Baloglu, S. y K. McCleary (1999). "A Model of Destination Image Formation”. Annals of Tourism Research, 26 (4), 868-897.

Barroso, C., E. Martín y D. Martín (2007). “The Influence of Market Heterogeneity on the Relationship between a Destination's Image and Tourists' Future Behaviour". Tourism Management, 28 (1), 175-187.

Bigné, E., M. Sánchez y J. Sánchez (2001). “Tourism Image, Evaluation Variables and After Purchase Behaviour: Inter-Relationship”. Tourism Management, 22 (6), 607-616.

Boo, S., J. Busser y S. Baloglu (2009). "A Model of Customer-Based Brand Equity and its Application to Multiple Destinations". Tourism Management, 30 (2), 219-231.

Bornhorst, T., J. Ritchie y L. Sheehan (2010). "Determinants of Tourism Success for DMo \& Destinations: An Empirical Examination of Stakeholders' Perspectives”. Tourism Management, 31 (5), 572-589.

Bowen, J. y S. Chen (2001). "The Relationship Between Customer Loyalty and Customer Satisfaction”. International Journal of Contemporary Hospitality Management, 13 (5), 213-217.

Cals, J., J. Capella y E. Vaqué (1997). Gestió Pública del Turisme. Manual per les Administracions Locals de les zones d'Interior, Barcelona: Fundació Carles Pi i Sunyer. 
Caruana, A., H. Money y P. Berthon (2000). "Service Quality and Satisfaction - The Moderating Role of Value”. European Journal of Marketing, 34 (11-12), 1338-1352.

Chen, C. y F. Chen (2010). “Experience Quality, Perceived Value, Satisfaction and Behavioral Intentions for Heritage Tourists”. Tourism Management, 31 (1), 29-35.

Chen, C. y D. Tsai (2007). "How Destination Image and Evaluative Factors Affect Behavioral Intentions?” Tourism Management, 28 (4), 1115-1122.

Chi, C. y H. Qu (2008). "Examining the Structural Relationships of Destination Image, Tourist Satisfaction and Destination Loyalty: An Integrated Approach”. Tourism Management, 29 (4), 624-636.

Chon, K. (1989). “Understanding Recreational Travelers' Motivation, Attitude and Satisfaction”. Tourism Review, 44 (1), 3-7.

Conde, E. et al. (2010). Posicionamiento turístico del estado de Colima. Buenos Aires: El Aleph.

Consejo Nacional Empresarial Turístico. (2011). Panorama de la Actividad Turística en México, núm. 5. Universidad Anáhuac. Disponible en < http://www.cnet.org.mx/sites/default/files/panorama_5.pdf > [diciembre de 2012].

Crompton, J. (1979). "Motivations of Pleasure Vacation”. Annals of Tourism Research, 6 (4), 408-424.

------ (1992). "Structure of Vacation Destination Choice Sets”. Annals of Tourism Research, 19 (3), 420-434.

Echtner, C. y J. Ritchie (2003). “The Meaning and Measurement of Destination Image”. The Journal of Tourism Studies, 14 (1), 37-46.

Elbe, J., L. Hallén y B. Axelsson (2009). "The Destination-Management Organisation and the Integrative Destination-Marketing Process”. International Journal of Tourism Research, 11 (3), 283-296.

Gallarza, M., I. Gil y H. Calderón (2002). “Destination Image. Towards a Conceptual Framework”. Annals of Tourism Research, 29 (1), 56-78.

Gartner, W. y M. Konecnik (2011). "Tourism Destination Brand Equity Dimensions: Renewal versus Repeat Market”. Journal of Travel Research, 50 (5), 471-481.

Gobierno de los Estados Unidos Mexicanos. (2011). $5^{\circ}$ Informe de Gobierno. 
"Anexo estadístico". México: Presidencia de la República. Disponible en: < http://biblioteca.itam.mx/docs/infgob11/AnexoEstadisticoPDF/ anexo \%20ompleto.pdf > . [diciembre de 2012].

Gobierno del Estado de Colima (2010). “Anexo estadístico”, Primer informe de gobierno.

Gobierno del Estado de Colima (2011). "Anexo estadístico” Segundo informe de gobierno.

Gobierno del Estado de Colima (2012). “Anexo estadístico” Tercer informe de gobierno.

Gutiérrez, C. et al. (2010). "Calidad estética de las playas de Manzanillo, Colima, durante el 2007-2008”, en L. Ruelas et al. (eds.). Gobernanza ambiental para el manejo sustentable de recursos: La experiencia de Canadá en México. Xalapa: El Colegio de Veracruz.

Hallowell, R. (1996). “The Relationships of Customer Satisfaction, Customer Loyalty, and Profitability: An Empirical Study”. International Journal of Service Industry Management, 7 (4), 27-42.

Hu, H., J. Kandampully y T. Juwaheer (2009). "Relationships and Impacts of Service Quality, Perceived Value, Customer Satisfaction, and Image: An Empirical Study”. The Service Industries Journal, 29 (2), 111-125. INEGI (2011). "Manzanillo, Colima”, en México en cifras. Información Nacional, por entidad federativa y municipios [en línea]. Instituto Nacional de Estadística y Geografía. Disponible en: http://www3.inegi. org.mx/sistemas/mexicocifras [2012, noviembre].

------ (2013). Calculadora de inflación [en línea]. Disponible en: http:// www.inegi.org.mx/sistemas/indiceprecios/CalculadoraInflacion. aspx [2013, enero].

Íñiguez, L. et al. (2007). "La gestión integral en playas turísticas: herramientas para la competitividad”. Gaceta Ecológica, 82 (1), 77-83.

Konecnik, M. y W. Gartner (2007). "Customer-Based Brand Equity for a Destination”. Annals of Tourism Research, 34 (2), 400-421.

Kotler, P., J. Bowen y J. Makens (1993). Marketing para turismo. Madrid: Prentice Hall.

Kotler, P. y D. Gertner (2004). "Country as Brand, Product and Beyond: A Place Marketing and Brand Management Perspective”, en N. Mor- 
gan, A. Pritchard y R. Pride (eds.), Destination Branding, Creating the Unique Destination Proposition. Oxford: Elsevier ButterworthHeinemann, 40-56.

Lee, Ch., Y. Yoon y S. Lee (2007). "Investigating the Relationships among Perceived Value, Satisfaction and Recommendations: The Case of the Korean DMz”. Tourism Management, 28 (1), 204-214.

Lundberg, D., M. Krishnamoorthy y M. Stavenga (1995). Tourism Economics. Nueva York: John Wiley \& Sons.

Miralbell, O. (2007). Gestión de oficinas de turismo. Barcelona: Editorial de la Universitat Oberta de Catalunya.

Murdaugh, H. (ed.) (2005). Fundamentals of Destination Management and Marketing. Lansing: Educational Institute of the American Hotel \& Lodging Association.

Okello, M. y S. Yerian (2009). "Tourist Satisfaction in Relation to Attractions and Implications for Conservation in the Protected Areas of the Northern Circuit, Tanzania”. Journal of Sustainable Tourism, 17 (5), 605-625.

Oliver, R. (1999). “Whence Consumer Loyalty”. Journal of Marketing, 63, 33-44.

Oppermann, M. (1999). "Predicting Destination Choice - A Discussion of Destination Loyalty”. Journal of Vacation Marketing, 5 (1), 51-65.

------ (2000). “Tourism Destination Loyalty”. Journal of Travel Research, 39

(1), 78-84.

Organización Mundial del Turismo. (2007). A practical guide to Tourism Destination Management. Madrid: Organización Mundial del Turismo.

Parasuraman, A., V. Zeithaml y L. Berry (1988). "SERVQUAL: A MultipleItem Scale for Measuring Consumer Perceptions of Service Quality”. Journal of Retailing, 64 (1), 12-40.

Pike, S. (2002). "Destination Image Analysis - A Review of 142 Papers from 1973 to 2000”. Tourism Management, 23 (5), 541-549.

------ (2004). Destinations Marketing Organizations. Ámsterdam: Elsevier.

------ (2005). “Tourism Destination Branding Complexity”. Journal of Product \& Brand Management, 14 (4), 258-259. (2009). "Destination Brand Positions of a Competitive Set of Near- 
Home Destinations”. Tourism Management, 30 (6), 857-866.

Pike, S., Bianchi, C., Kerr, G. y Patti, C. (2010). “Consumer-based brand equity for Australia as a long-haul tourism destination in an emerging market”. International Marketing Review, 27, (4): 434-449.

Pizam, A. y A. Milman (1993). "Predicting Satisfaction among First Time Visitors to a Destination by Using the Expectancy Disconfirmation Theory”. International Journal of Hospitality Management, 12 (2), 197-209.

Presidencia de la República (2011). $5^{\circ}$ Informe de Gobierno. Anexo estadístico [en línea]. México: Presidencia de la República. Disponible en: http:// biblioteca.itam.mx/docs/infgob11/AnexoEstadisticoPDF/anexo \% 20 ompleto.pdf [2012, diciembre].

Presenza, A., L. Sheehan y J. Ritchie (2005). “Towards a Model of the Roles and Activities of Destination Management Organizations". Journal of Hospitality, Tourism and Leisure Science, 3 (1), 1-16.

Ritchie, B. y G. Crouch (2005). The Competitive Destination. A Sustainable Tourism Perspective. Trowbridge: CABI Publishing.

Sánchez, J. et al. (2006). "Perceived Value of the Purchase of a Tourism Product”. Tourism Management, 27 (6), 394-409.

Sectur (2000). Competitividad y Desarrollo de Productos Turísticos Exitosos. México: Secretaría de Turismo (Documentos Técnicos de Competitividad, fascículo V).

Simpson, P. y J. Siguaw (2008). "Destination Word of Mouth: The Role of Traveler Type, Residents, and Identity Salience”. Journal of Travel Research, 47 (2), 167-182.

Valls, J. et al. (2004). Gestión de destinos turísticos sostenibles. Barcelona: Ediciones Gestión 2000.

Williams, P. y G. Soutar (2009). "Value, Satisfaction and Behavioral Intentions in an Adventure Tourism Context". Annals of Tourism Research, 36 (3), 413-438.

World Tourism Organisation y European Travel Comission. (2009). Handbook on Tourism Destination Branding. Madrid: World Tourism Organisation.

138 World Tourism Organisation (2010). Destination Management, Conceptual 
Framework. <http://www.unwto.org/destination/conceptual/ > [marzo de 2010].

Yang, Z. y R. Peterson (2004). "Customer Perceived Value, Satisfaction, and Loyalty: The Role of Switching Costs". Psychology and Marketing, 21 (10), 799-822.

Yoon, Y. y M. Uysal (2005). "An Examination of the Effects of Motivation and Satisfaction on Destination Loyalty: A Structural Model". Tourism Management, 26 (1), 45-56.

Yuan, J. y S. Jang (2008). "The Effects of Quality and Satisfaction on Awareness and Behavioral Intentions: Exploring the Role of a Wine Festival". Journal of Travel Research, 46 (3), 279-288. 